\title{
MLL Partial Tandem Duplication
}

National Cancer Institute

\section{Source}

National Cancer Institute. MLL Partial Tandem Duplication. NCI Thesaurus. Code

C113507.

A chromosomal rearrangement where exons 5 through 11 or 5 through 12 of the MLL gene are duplicated and inserted upstream of exon 4 of the gene. This duplication results in transcripts and proteins that have the $\mathrm{N}$-terminal region, which contains the AT hook DNA-binding motifs, and the transcriptional repression domain duplicated. This mutation is associated with myelodysplastic syndrome (MDS) and susceptibility of MDS progression to acute myeloid leukemia. 